\title{
Impresión 3D por modelado por deposición fundida: Manejo, funcionamiento y aplicaciones biomédicas
}

\author{
3D printing by fused deposition modelling: Handling, operation and biomedical applications
}

Fecha de recepción y aceptación: 17 de febrero de 2021 y 29 de abril de 2021

DOI: $10.46583 /$ nereis_2021.13.809

\author{
Alba Cano Vicent ${ }^{1}$ y Ángel Serrano Aroca ${ }^{2 *}$ \\ ${ }^{1}$ Escuela de Doctorado. Biomaterials and Bioengineering Lab. Centro de Investigación Traslacional San Alberto Magno. Universidad \\ Católica de Valencia San Vicente Mártir. \\ ${ }^{2}$ Biomaterials and Bioengineering Lab. Centro de Investigación Traslacional San Alberto Magno. Universidad Católica de Valencia \\ San Vicente Mártir. \\ * Correspondencia: Universidad Católica de Valencia San Vicente Mártir. Centro de Investigación Trasnacional San Alberto Magno. \\ Calle Guillem de Castro 94. 46001 Valencia. España.E-mail: angel.serrano@ucv.es
}

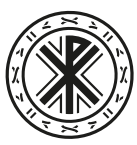

Universidad Católica de Valencia San Vicente Mártir

\section{RESUMEN}

El desarrollo de la impresión 3D está creciendo de forma exponencial debido a la posibilidad única que proporciona de poder fabricar piezas a medida de forma reproducible y personalizada. Aunque todavía está lejos de su desarrollo óptimo debido a la todavía lenta velocidad de impresión y a las limitaciones que supone el no poder disponer de todo tipo de material para imprimir en 3D, esta tecnología está abarcando áreas de aplicación de todo tipo que van desde la construcción de edificios hasta el desarrollo de órganos. Dentro de todas las técnicas de impresión 3D desarrolladas, el modelado por deposición fundida (FDM) es una de las más utilizadas y permite construir piezas avanzadas a partir del diseño asistido por ordenador. En este artículo se exponen todos los conceptos básicos necesarios para su manejo y funcionamiento de la impresión 3D por FDM, los tipos de filamentos y su aplicación avanzada en biomedicina como la impresión 3D de soportes porosos (scaffolds en inglés) para ingeniería tisular y la bioimpresión de células combinadas con biomateriales.

PALABRAS CLAVE: impresión 3D, modelado por deposición fundida, biomateriales, biomedicina, scaffolds, bioimpresión, medicina regenerativa.

\section{ABSTRACT}

The development of 3D printing is growing exponentially due to their unique characteristics. Thus, this technique is capable of fabricating custom pieces in a reproducible and personalized way. Although it is still far from its optimal development due to the still slow printing speed and the limitations of materials available for 3D printing in the market currently, this technology is constantly broadening its application areas which covers from building construction to organ fabrication. Among all the 3D printing techniques developed so far, fused deposition modeling (FDM) is one of the most common and it allows the construction of advanced pieces from computer-aided design. This article presents all the basic concepts necessary for the handling and 
operation of a FDM 3D printer, the types of filaments and the advanced applications of 3D printing in biomedicine such as the fabrication of scaffolds for tissue engineering and the bioprinting of cells combined with biomaterials.

KEYWORDS: 3D printing, fused deposition modeling, biomaterials, biomedicine, scaffolds, bioprinting, regenerative medicine.

\section{INTRODUCCIÓN}

La impresión 3D, en los últimos años, ha sufrido una gran evolución que ha propiciado que su uso esté actualmente en crecimiento exponencial. La impresión 3D inicialmente se empleaba para hacer moldes o prototipos [1]. Tras ello, aumentó el uso de la impresión 3D debido a la capacidad de diseño más preciso y reproducible de los modelos. De esta manera, se mejoró la rapidez con la que se conseguían los diseños y se posibilitó la realización de prototipos o diseños con diferentes tamaños, estilos, materiales, colores, etc. [2] Actualmente, la impresión 3D ha llegado al público en general, ya que se puede adquirir una impresora 3D incluso en un centro comercial. Cualquier persona hoy en día puede diseñar prototipos e imprimirlos directamente en su casa. Todo esta demanda ha favorecido a pasos agigantados el desarrollo de múltiples técnicas de impresión 3D [3].

Entre todas las técnicas de impresión 3D que existen, cabe destacar el modelado por deposición fundida, la impresión directa, la impresión por inyección, el sinterizado selectivo por láser, entre otras [4]. De todas estas técnicas desarrolladas, una de las más utilizadas es la impresión 3D por modelado por deposición fundida (FDM), que es una técnica de tecnología avanzada controlada por el ordenador de prototipo rápido (PR). Gracias a esta técnica avanzada, es posible producir piezas constituidas de materiales porosos a través del método de fabricación de capas [5]. El PR de impresión 3D se caracteriza porque, generalmente, comprende el diseño del modelo usando un software de diseño 3D, el cual se expresa en una serie de laminaciones o capas. De esta forma, la impresora 3D reproduce el diseño capa a capa, una encima de otra, hasta obtener el modelo completo. El prototipo rápido FDM tiene ventajas como el perfecto control de la arquitectura de la matriz (forma, tamaño, ramificación, geometría, interconectividad y orientación) produciendo una estructura biomimética, que varía en diseño y composición según el material utilizado.

En esta publicación se muestra la relevancia de la impresión 3D, más concretamente, conceptos básicos en el manejo de la impresión 3D por modelado por deposición fundida y aplicaciones avanzadas como la impresión 3D de soportes porosos (scaffolds en inglés) para ingeniería tisular.

\section{ETAPAS PRINCIPALES EN LA IMPRESIÓN 3D POR FDM}

Como esquema general, la impresión 3D por FDM empieza con el diseño virtual de la pieza que se quiere imprimir. Para ello, se utiliza un software CAD (diseño asistido por computadora) y se obtiene un archivo en formato ".stl”. Algunos de los softwares más utilizados son: FreeCAD, Autodesk Inventor o Tinkercad. A continuación, con un programa de corte o laminación se procesa este archivo 
“.stl” para convertir el diseño en instrucciones específicas para la impresora, de modo que estar pueda “entender” el diseño. Además, se añaden las especificaciones necesarias para poder imprimir la pieza, como velocidad de impresión, tamaño del hilo de impresión, temperatura necesaria, altura de las capas, etc. En este caso, los programas más comunes que se utilizan son Slic3r y Cura, que son de libre acceso. Así, se obtiene un archivo con formato ".gcode", que ya puede ser procesado por la impresora para obtener la pieza [6]. En la figura 1, se muestra un esquema general del proceso de impresión 3D por FDM.

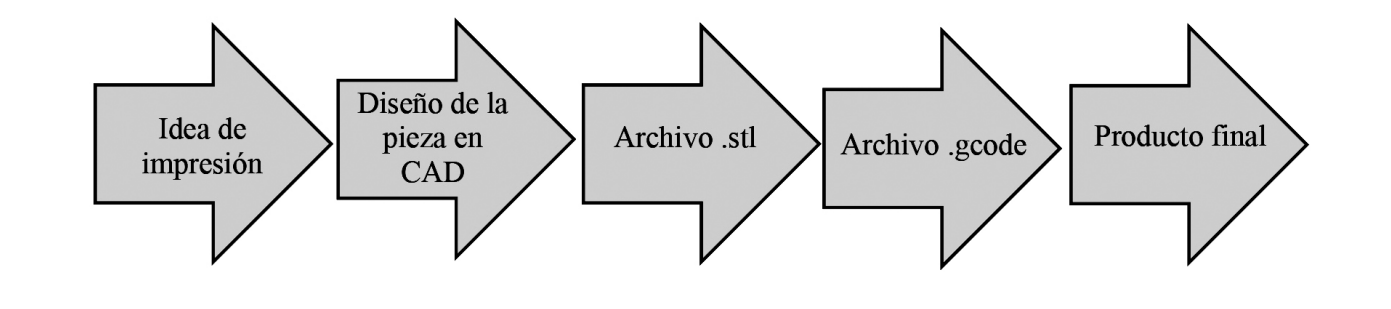

Fig. 1. Esquema general del proceso de impresión 3D por FDM con sus etapas principales. Fuente: elaboración propia.

La norma ISO / ASTM 52901: 2017 - Additive Manufacturing - General Principles define los principios generales, especifica los requisitos y brinda pautas y aplicabilidad para su uso como base para obtener piezas hechas a través de la fabricación aditiva, incluida la FDM.

\section{FUNCIONAMIENTO DE UNA IMPRESORA 3D POR FDM}

La técnica de impresión 3D por FDM consiste en la deposición capa por capa de material polimérico fundido, una encima de otra, hasta completar la pieza diseñada. Es decir, consiste en la utilización de un filamento polimérico, que se encuentra en una bobina, y se funde, a medida que va pasando por el extrusor, cuando alcanza el punto de fusión [7]. El extrusor se desplaza en los ejes X e Y con el fin de ir dando forma a cada una de las capas que se van formando. A su vez, la cama se mueve en el eje $Z$, descendiendo un nivel cada vez que se ha formado una nueva capa.

Más concretamente, el extrusor es la pieza donde se produce el calentamiento del filamento hasta la temperatura de fusión para poder formar la pieza diseñada, proceso también denominado de extrusión. Esta parte de la impresora 3D consta a su vez de una serie de componentes, como el motor de extrusión, encargado de proporcionar la potencia para arrastrar el filamento y fundirlo para poder depositarlo capa a capa en la cama, es decir, el lugar de la impresora 3D donde la pieza se deposita que puede estar calefactado o no. Además, la impresora 3D consta de una polea y una palanca, que, gracias a sus dientes, presionan el filamento y lo arrastran hasta la punta del extrusor (o hot-end en inglés). Así mismo, el sistema dispone de un ventilador, que permite refrigerar el extrusor, y del tubo de Fibonacci, que se encuentra en la entrada del extrusor e impide que se produzcan roturas en el filamento. Por último, en la salida, como se ha comentado, se encuentra el hot-end, que es la pieza encargada de fundir el material polimérico para poder extrudirlo [8] (véase figura 2). 


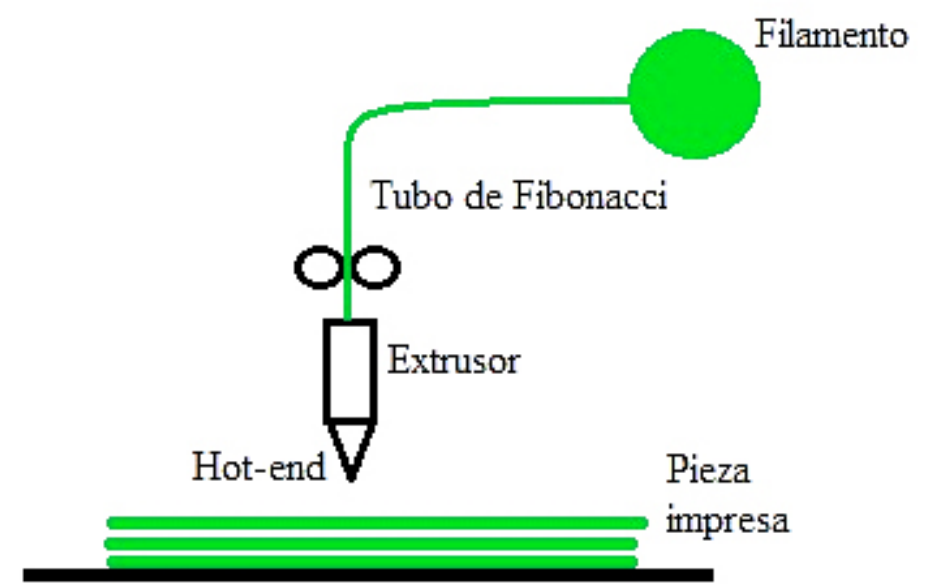

Base de impresión (cámara calefactada)

Fig. 2. Esquema básico del funcionamiento de una impresora 3D por modelado por deposición fundida. Fuente: elaboración propia.

El extrusor se encuentra colocado en un carro que permite que se mueva en las direcciones X e Y. De esta manera, a medida que va generándose la pieza, el extrusor se mueve para ir dando forma al diseño.

La otra gran parte importante de la impresora es la base de impresión o cama, que consta de un cristal grueso que sirve de soporte para la impresión y suele ser de forma cuadrada o rectangular. Existen dos tipos de bases de impresión: frías o calientes. Las bases de impresión calientes, también denominadas camas calientes, están calefactadas para poder alcanzar cierta temperatura con el fin de que la diferencia entre el hot-end y la cama de impresión no sea tan grande cuando el material fundido caiga sobre ella. De esta manera, se puede evitar que se produzcan imperfecciones como las roturas en la pieza impresa por el cambio de temperatura, o el conocido como warping en inglés, que se produce cuando la primera capa de una pieza se deforma y se despega de la cama de impresión [9]. El warping suele producirse sobre todo en las piezas grandes porque hay diferencias térmicas más significativas que hacen que las piezas acaben despegándose por las esquinas.

\section{TIPOS DE FILAMENTOS}

A continuación, se exponen algunos ejemplos de tipos de filamentos utilizados en la impresión 3D por FDM, como son el ácido poliláctico (PLA), el acrilonitrilo butadieno estireno (ABS), el polivinil alcohol (PVA), el elastómero termoplástico (TPE), el policarbonato (PC) y el ácido poliláctico con grafeno (figura 3). 


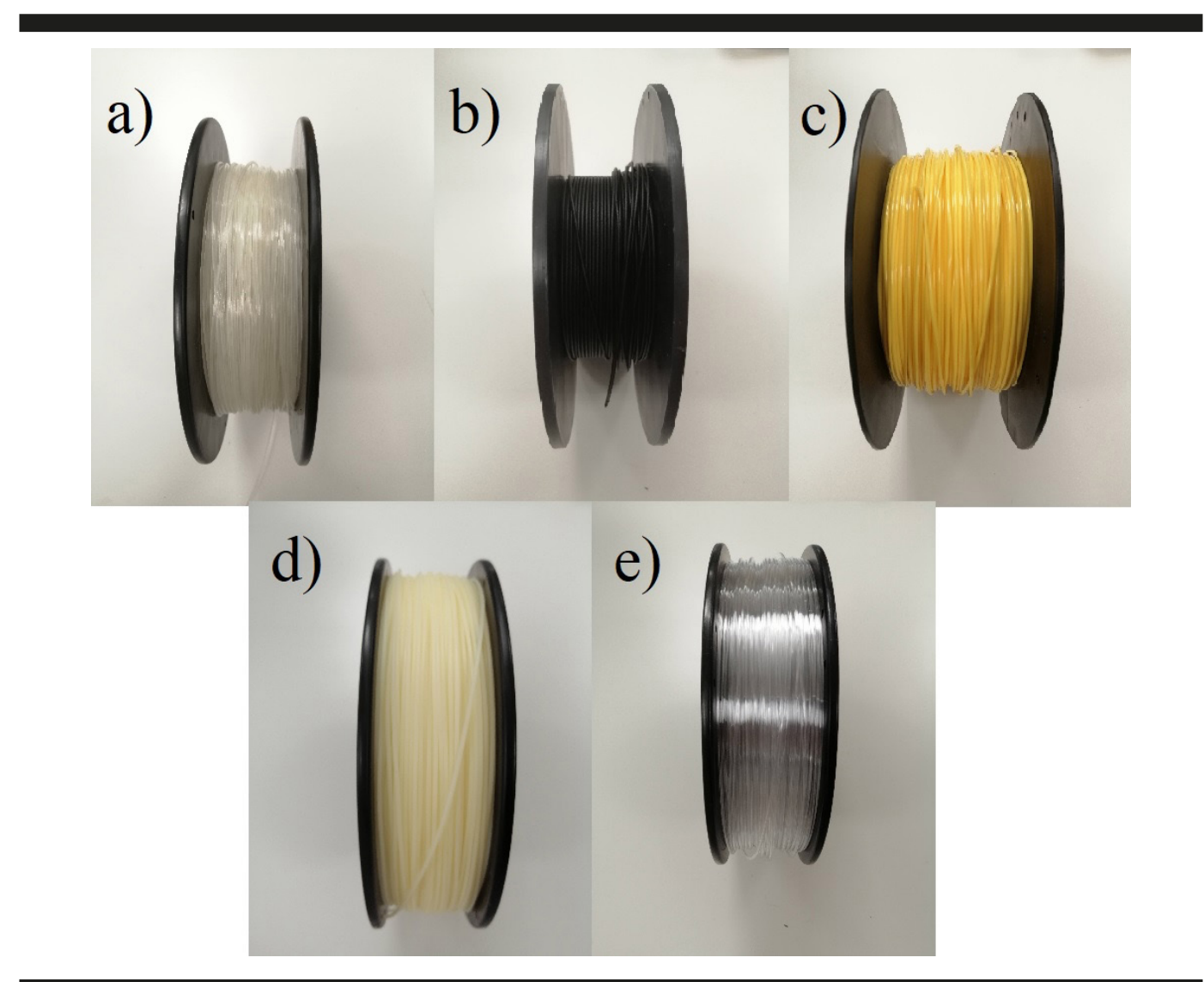

Fig. 3. Ejemplo de tipos de filamentos disponibles: a) elastómero termoplástico; b) ácido poliláctico con grafeno; c) PLA; d) PVA; e) policarbonato. Fuente: elaboración propia.

\section{Ácido poliláctico}

El PLA es un plástico biodegradable que proviene de la fermentación de cultivos como el maíz o la patata [10]. Se imprime a una temperatura de $220^{\circ} \mathrm{C}$ y no necesita cama caliente. Es duro y fuerte, aunque no es muy resistente [11]. No es tóxico. Debido a todas sus propiedades, las aplicaciones que posee son infinitas, incluida su utilización para aplicaciones avanzadas como la ingeniería tisular [12] $\mathrm{y}$ en biosensores [13].

\section{Acrilonitrilo butadieno estireno}

El ABS fue uno de los primeros materiales que se desarrolló para la impresión 3D. Es resistente al desgaste, al impacto, soporta grandes temperaturas y tiene un bajo coste [10]. El gran inconveniente de este material es que se funde a temperaturas entre $240-250{ }^{\circ} \mathrm{C}$, por lo que necesita cama caliente para evitar problemas de warping y, además, proviene del petróleo. Al igual que con el PLA, posee muchas aplicaciones como la fabricación de microdispositivos [14] y microfluidos [15]. 


\section{Polivinil alcohol}

El PVA es material hidrosoluble, es decir, se disuelve al estar en contacto con el agua. Se imprime a temperatura parecida al PLA $\left(\sim 220^{\circ} \mathrm{C}\right)$ por lo que se suele utilizar como soporte, para después poder disolverlo con facilidad [10]. Además, gracias a sus propiedades, posee otras aplicaciones como su uso para poder hacer "maquetas" dentales [16]. El PVA se utiliza en gran medida en estado entrecruzado, en aplicaciones biomédicas como un hidrogel en la bioimpresión, es decir, la impresión 3D de biomaterial combinado con células en medicina regenerativa [17].

\section{Elastómero termoplástico}

El TPE es un material flexible que se deforma muy fácilmente, por lo que puede contrarrestar cualquier tipo de carga sobre él. Se imprime a $230{ }^{\circ} \mathrm{C}$ y no es necesario el uso de cama caliente [18]. Debido a su gran flexibilidad, el filamento debe ir directamente enganchado al extrusor, sin pasar por el tubo de Fibonacci [19]. Sus características han hecho que este filamento revolucione la industria textil y, gracias a él, sea posible imprimir ropa [20]. También se han desarrollado plantillas ortopédicas de TPE [21].

\section{Policarbonato}

El PC es un material transparente que resiste muy altas temperaturas, ligeramente flexible y muy resistente al impacto[10]. El mayor inconveniente es que es necesario imprimirlo a más de $280{ }^{\circ} \mathrm{C}$, por lo que necesita una cama caliente a temperatura muy alta, entre $80-100{ }^{\circ} \mathrm{C}$. Pese a esta desventaja, su aplicación es cada vez mayor debido a sus excelentes propiedades. Se utiliza para aplicaciones ortopédicas, dentales [22] e ingeniería tisular [23].

\section{Ácido poliláctico con grafeno}

El grafeno es uno de los materiales que más recientemente se ha incluido en la impresión 3D. Debido a que el grafeno se utiliza en impresión 3D habitualmente mezclado en baja concentración con PLA, se imprime a una temperatura parecida al PLA, sobre $220^{\circ} \mathrm{C}$. Sin embargo, en este caso es necesario el uso de cama caliente, debido a las propiedades específicas del grafeno. Su característica principal es su excelente conductividad térmica y eléctrica que proporciona filamentos conductores. Esta propiedad conductora de la electricidad le otorga la posibilidad de poder aplicar electricidad que estimule la proliferación o diferenciación celular en soportes porosos para ingeniería tisular [24]. Además, es más resistente que filamentos más comunes como el ABS o el PLA [25]. Entre sus aplicaciones, destacan las herramientas quirúrgicas [26], biomateriales neurogénicos [27], blindaje por inducción electromagnética [28], etc. 
Tabla 1. Propiedades y aplicaciones más importantes de los filamentos de impresión 3D

\begin{tabular}{|c|c|c|c|c|}
\hline & $\mathrm{T}^{\mathrm{o}}$ impresión & Pros & Contras & Aplicaciones \\
\hline PLA & $220^{\circ} \mathrm{C}[11]$ & $\begin{array}{l}\text { Biodegradable [10], no es } \\
\text { tóxico, bajo coste [11] }\end{array}$ & No muy resistente [11] & $\begin{array}{l}\text { Ingeniería tisular [12], } \\
\text { biosensores [13] }\end{array}$ \\
\hline ABS & $240-250^{\circ} \mathrm{C}[10]$ & Resistente [10] & $\begin{array}{l}\text { Necesita alta temperatura } \\
\text { y cama caliente, tóxico }\end{array}$ & $\begin{array}{l}\text { Microdispositivos [14], } \\
\text { biomedicina[15] }\end{array}$ \\
\hline PVA & $210-220^{\circ} \mathrm{C}[10]$ & $\begin{array}{l}\text { Hidrosoluble, útil } \\
\text { como soporte [10] }\end{array}$ & $\begin{array}{l}\text { Le afecta mucho la } \\
\text { humedad [10] }\end{array}$ & $\begin{array}{l}\text { Soporte, maquetas } \\
\text { dentales [16] }\end{array}$ \\
\hline TPE & $210^{\circ} \mathrm{C}[18]$ & Flexible [18] & No soporta la humedad [19] & $\begin{array}{l}\text { Textil [20], plantillas } \\
\text { ortopédicas [21] }\end{array}$ \\
\hline $\mathrm{PC}$ & $280^{\circ} \mathrm{C}[10]$ & $\begin{array}{l}\text { Muy resistente a golpes y } \\
\text { a altas temperaturas [10] }\end{array}$ & $\begin{array}{l}\text { Necesita alta temperatura } \\
\text { y cama caliente }[10]\end{array}$ & $\begin{array}{l}\text { Dental, ortopédico [22], } \\
\text { ingeniería tisular [23]. }\end{array}$ \\
\hline PLA/grafeno & $220^{\circ} \mathrm{C}$ & $\begin{array}{l}\text { Conductividad, resistente } \\
\text { [25]Stereolithography (SLA }\end{array}$ & $\begin{array}{l}\text { Necesita cama caliente, } \\
\text { difícil de imprimir }\end{array}$ & $\begin{array}{l}\text { Herramientas quirúrgicas [26], } \\
\text { biomateriales neurogénicos } \\
\text { [27], blindaje por inducción } \\
\text { electromagnética [28] }\end{array}$ \\
\hline
\end{tabular}

\section{APLICACIONES AVANZADAS DE LA IMPRESIÓN 3D}

El rápido desarrollo de la impresión 3D ha hecho que cada vez sus aplicaciones sean mayores. Debido a que los filamentos están constituidos de polímeros que presentan con frecuencia propiedades excelentes de biocompatilidad, la impresión 3D presenta gran potencial para ser utilizada en aplicaciones biomédicas. Además de su biocompatibilidad, otras características que hacen ideal su aplicación como biomateriales son, entre otras, su buen comportamiento mecánico y estructural [29]. Todas estas propiedades confieren a los materiales desarrollados que la impresión 3D sea ideal para fabricar scaffolds que actúan como matriz extracelular (ECM) artificial en la ingeniería de tejidos (figura 4).

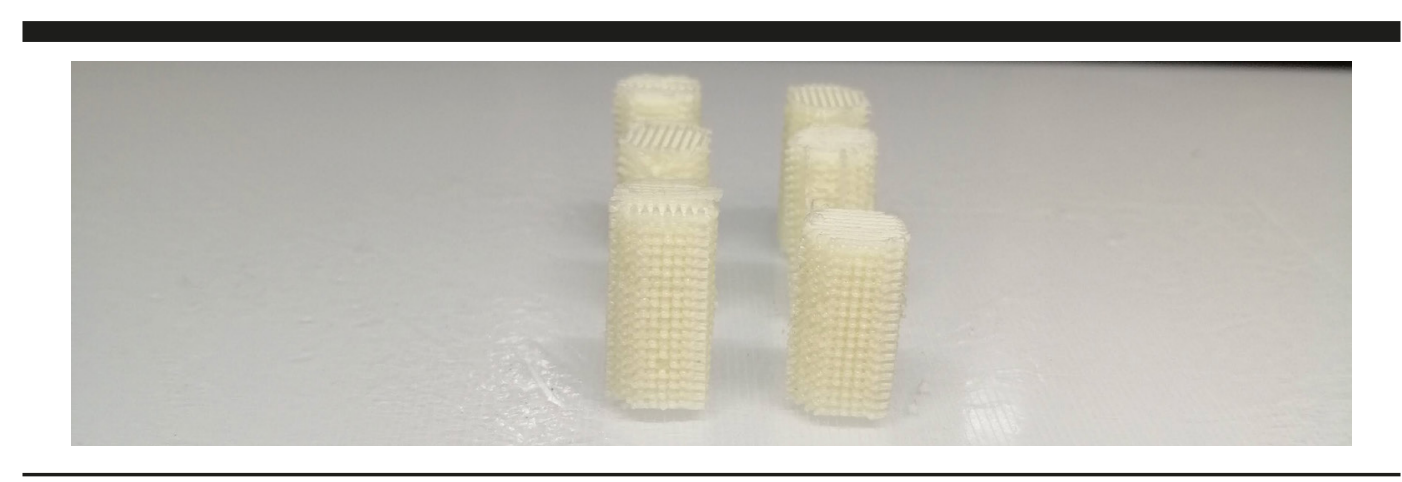

Fig. 4. Impresión de un scaffold de PVA mediante impresión 3D por FDM. Fuente: elaboración propia.

En este caso, el papel de los biomateriales es proporcionar un soporte celular 3D que controle y guíe la regeneración localizada del tejido proporcionando microambientes celulares potencialmente 
controlables similares a los in vivo [30]. Los scaffolds se utilizan como soportes para el crecimiento de tejido óseo, cartílago, ligamentos, piel, vasos sanguíneos, nervios y músculo [31]. Cuando los scaffolds son biodegradables, estos soportes porosos permiten que el material se desintegre al mismo tiempo que se va generando nuevo tejido y así poder producir por bioingeniería incluso órganos completos [32].

Las tecnologías tradicionales no tienen la habilidad de incorporar arquitectura interna y controlar la porosidad de los scaffolds. Sin embargo, con la impresión 3D por modelado de deposición fundida es posible controlar el tamaño y la distribución de los poros [33]. Asimismo, gracias a la tecnología 3D es posible reproducir las propiedades mecánicas necesarias para poder favorecer la regeneración tisular [34]. Para la fabricación de scaffolds por impresión 3D, se mezclan diferentes biomateriales con el fin de conseguir aprovechar las mejores propiedades de cada uno de ellos. Por ejemplo, Lee et al. desarrollaron un scaffold, con la tecnología de impresión por modelado de deposición fundida, de hidroxiapatita y policaprolactona (PCL). Este scaffold, que favorece la regeneración ósea, se probó en conejos, favoreciendo la regeneración del cartílago, el cual tenía propiedades histológicas comparables a las del cartílago normal [35]. Además, dependiendo del tipo de aplicación, el scaffold diseñado puede requerir propiedades físico-químicas específicas, como biodegradabilidad o propiedades mecánicas; y propiedades morfológicas, como topología de superficie, tamaño del poro, etc., para mimetizar el ambiente celular in vivo según la aplicación concreta de regeneración [36]. La mayoría de los biomateriales empleados en impresión 3D se pueden utilizar para la liberación de biomoléculas como antibióticos, factores de crecimiento, biometales, etc. [37]. Esta ventaja supone un gran avance en la industria farmacéutica, dado que la liberación controlada de fármacos permite la continua administración de un fármaco de forma eficaz [38]. La impresión 3D ha permitido el desarrollo de implantes capaces de liberar incluso tres tratamientos diferentes (captopril, nifedipina, glipizida) de forma dosificada [39]. Por otra parte, gracias a los avances que se han ido produciendo en la tecnología de la impresión 3D, es posible crear organoides y modelos de laboratorio altamente complejos de bioingeniería producidos por bioprinting [40]. Mediante la bioimpresión, muchos laboratorios han creado modelos biológicamente funcionales compuestos por una amplia variedad de tejidos como neuronas [41], riñón [42], músculo esquelético [43], hígado [44], etc.

Por último, cabe mencionar que a pesar de que la mayoría de las herramientas quirúrgicas sirven para una amplia variedad de pacientes, algunas operaciones pueden requerir la utilización de instrumentos más singulares [45]. De esta manera, gracias a la unión de los avances en las tecnologías radiológicas, para poder tomar imágenes de la anatomía del paciente, y los avances en la tecnología de impresión 3D es posible obtener herramientas personalizadas de una forma más controlada y simplificada [46]. El hecho de poder fabricar herramientas quirúrgicas eficaces con la impresión 3D también favorece a los países subdesarrollados, pues puede conseguir reducir el coste hasta diez veces [47].

\section{CONCLUSIONES}

La impresión 3D ha sido pronosticada como la futura revolución industrial. Esta tecnología posee bajo coste de los materiales y características únicas como la capacidad precisa de diseño, su alta reproducibilidad, que permite poder realizar duplicados y poder obtener el diseño de un prototipo 
pudiendo cambiar muy fácilmente su color, forma, tamaño, estilo o material. Dentro de la impresión 3D, la técnica del modelado por deposición fundida (FDM) es la técnica más comúnmente utilizada. Dentro de la biomedicina, se está utilizando la impresión 3D por FDM para la fabricación de scaffolds que favorecen la regeneración tisular de todo tipo de tejido. Además, la impresión 3D de órganos, conocida como bioimpresión, está avanzando a un elevado ritmo augurando un futuro muy prometedor para el ser humano. Por este motivo, muchos grupos de investigación de todo el mundo están trabajando para poder superar todas las limitaciones que supone la creación de tejidos y órganos completos donde debe haber un equilibrio entre proporcionar un soporte estructural al modelo impreso y la creación de un entorno viable para las células que varía según la aplicación biomédica.

\section{AGRADECIMIENTOS}

Los autores agradecen a la Fundación Universidad Católica de Valencia San Vicente Mártir su apoyo económico a través de los proyectos 2019-231-001UCV y 2020-231-001UCV (otorgados a Á. S-A.).

\section{REFERENCIAS BIBLIOGRÁFICAS}

[1] Wickramasinghe S, Do T, Tran P. FDM-Based 3D printing of polymer and associated composite: A review on mechanical properties, defects and treatments. Vol. 12, Polymers. MDPI AG; 2020, pp. 1-42.

[2] Berman B. 3-D printing: The new industrial revolution. Bus Horiz. 2012 Mar;55(2):155-62.

[3] Fudos I, Ntousia M, Stamati V, Charalampous P, Kontodina T, Kostavelis I, et al. A Characterization of 3D Printability. In CAD Solutions, LLC; 2020, pp. 363-7.

[4] Bekas DG, Hou Y, Liu Y, Panesar A. 3D printing to enable multifunctionality in polymer-based composites: A review. Vol. 179, Composites Part B: Engineering. Elsevier Ltd; 2019, p. 107540.

[5] Nkomo N, Gwamuri J, Roselyn N, Ndebele S, Nkiwane L, Nkomo NZ, et al. A Study of Applications of 3D printing technology and potential applications in the plastic thermoforming industry. Vol. 07, International organization of Scientific Research. 2017 [consultado: 26 enero 2021]. Disponible en: www.iosrjen.org

[6] Ortiz-Acosta D, Moore T. Functional 3D Printed Polymeric Materials. En: Functional Materials. IntechOpen; 2019.

[7] Saroia J, Wang Y, Wei Q, Lei M, Li X, Guo Y, et al. A review on 3D printed matrix polymer composites: its potential and future challenges. Vol. 106, International Journal of Advanced Manufacturing Technology. Springer; 2020, pp. 1695-721.

[8] Chia HN, Wu BM. Recent advances in 3D printing of biomaterials. J Biol Eng. 2015 Mar 1;9(1):4 [consultado: 22 enero 2021]. Disponible en: http://www.jbioleng.org/content/9/1/4

[9] Ngo TD, Kashani A, Imbalzano G, Nguyen KTQ, Hui D. Additive manufacturing (3D printing): A review of materials, methods, applications and challenges. Vol. 143, Composites Part B: Engineering. Elsevier Ltd; 2018, pp. 172-96.

[10] Singh H. A comprehensive study on 3D printing technology; 2020. 
[11] Aspler J, Kingsland A, Cormier LM, Zou X. 3D printing - A review of technologies, markets, and opportunities for the forest industry; 2016.

[12] Zuluaga F. Algunas aplicaciones del ácido poli-L-láctico. Rev la Acad Colomb ciencias exactas, físicas y Nat. 2013;37(142) [consultado: 25 enero 2021]. Disponible en: http://www.scielo.org. co/scielo.php?script=sci_arttext\&pid=S0370-39082013000100009

[13] Muñoz J, Pumera M. 3D-printed biosensors for electrochemical and optical applications. Vol. 128, TrAC - Trends in Analytical Chemistry. Elsevier B. V.; 2020, p. 115933.

[14] Li X, Cui R, Sun L, Aifantis KE, Fan Y, Feng Q, et al. 3D-printed biopolymers for tissue engineering application. Vol. 2014, International Journal of Polymer Science. Hindawi Publishing Corporation; 2014.

[15] Fornells E, Murray E, Waheed S, Morrin A, Diamond D, Paull B, et al. Integrated 3D printed heaters for microfluidic applications: Ammonium analysis within environmental water. Anal Chim Acta. 2020 Feb 15;1098:94-101.

[16] Muta S, Ikeda M, Nikaido T, Sayed M, Sadr A, Suzuki T, et al. Chairside fabrication of provisional crowns on FDM 3D-printed PVA model. J Prosthodont Res. 2020 Oct 1;64(4):4017 [consultado: 28 enero 2021]. Disponible en: https://linkinghub.elsevier.com/retrieve/pii/ S1883195819304293

[17] Bendtsen ST, Quinnell SP, Wei M. Development of a novel alginate-polyvinyl alcohol-hydroxyapatite hydrogel for 3D bioprinting bone tissue engineered scaffolds. J Biomed Mater Res Part A. 2017 May 1;105(5):1457-68 [consultado: 12 febrero 2021]. Disponible en: http://doi.wiley. com/10.1002/jbm.a.36036

[18] Hu X, Kang H, Li Y, Geng Y, Wang R, Zhang L. Preparation, morphology and superior performances of biobased thermoplastic elastomer by in situ dynamical vulcanization for 3D-printed materials. Polymer (Guildf). 2017 Jan 13;108:11-20.

[19] Georgopoulou A, Sebastian T, Clemens F. Thermoplastic elastomer composite filaments for strain sensing applications extruded with a fused deposition modelling 3D printer. Flex Print Electron. 2020 Sep 1;5(3):35002 [consultado: 28 enero 2021]. Disponible en: https://doi. org/10.1088/2058-8585/ab9a22

[20] Korger M, Bergschneider J, Lutz M, Mahltig B, Finsterbusch K, Rabe M. Possible applications of 3D printing technology on textile substrates. En: IOP Conference Series: Materials Science and Engineering. Institute of Physics Publishing; 2016, p. 012011 [consultado: 28 enero 2021]. Disponible en: https://iopscience.iop.org/article/10.1088/1757-899X/141/1/012011

[21] Mogan Y, Periyasamy R. Thermoplastic elastomer infill pattern impact on mechanical properties 3D printed customized orthotic insole. ARPN J Eng Appl Sci. 2016;11(10) [consultado: 28 enero 2021]. Disponible en: https://www.researchgate.net/publication/304887411

[22] Han X, Yang D, Yang C, Spintzyk S, Scheideler L, Li P, et al. Carbon Fiber Reinforced PEEK Composites Based on 3D-Printing Technology for Orthopedic and Dental Applications. J Clin Med. 2019 Feb 12;8(2):240 [consultado: 28 enero 2021]. Disponible en: http://www.mdpi. com/2077-0383/8/2/240

[23] Radhakrishnan S, Nagarajan S, Belaid H, Farha C, Iatsunskyi I, Coy E, et al. Fabrication of 3D printed antimicrobial polycaprolactone scaffolds for tissue engineering applications. Mater Sci Eng C. 2021 Jan 1;118. 
[24] Restrepo E, Restrepo M. Synergy between 3D Models and Tissue Engineering to Optimize Sinus Lift, Implant Placement and Immediate Loading in Partially Edentulous Patients. Artic Int J Oral Implantol Clin Res. 2013 [consultado: 11 febrero 2021]. Disponible en: https:/www. researchgate.net/publication/271261428

[25] Ponnamma D, Yin Y, Salim N, Parameswaranpillai J, Thomas S, Hameed N. Recent progress and multifunctional applications of 3D printed graphene nanocomposites. Vol. 204, Composites Part B: Engineering. Elsevier Ltd; 2021.

[26] Prashantha K, Roger F. Multifunctional properties of 3D printed poly(lactic acid)/graphene nanocomposites by fused deposition modeling. J Macromol Sci Part A Pure Appl Chem. 2017 Jan 2;54(1):24-9 [consultado: 28 enero 2021]. Disponible en: https://www.tandfonline.com/doi/ abs/10.1080/10601325.2017.1250311

[27] Jakus AE, Shah RN. Multi and mixed 3D-printing of graphene-hydroxyapatite hybrid materials for complex tissue engineering. J Biomed Mater Res Part A. 2017 Jan 1;105(1):274-83 [consultado: 28 enero 2021]. Disponible en: http://doi.wiley.com/10.1002/jbm.a.35684

[28] Sherrell PC, Mattevi C. Mesoscale design of multifunctional 3D graphene networks. Vol. 19, Materials Today. Elsevier B.V.; 2016, pp. 428-36.

[29] Wang X, Jiang M, Zhou Z, Gou J, Hui D. 3D printing of polymer matrix composites: A review and prospective. Vol. 110, Composites Part B: Engineering. Elsevier Ltd; 2017, pp. 442-58.

[30] Haider HK, Lei Y, Ashraf M. MyoCell, a cell-based, autologous skeletal myoblast therapy for the treatment of cardiovascular diseases. Curr Opin Mol Ther. 2008;10(6):611-21.

[31] Dong C, Lv Y. Application of collagen scaffold in tissue engineering: Recent advances and new perspectives. Vol. 8, Polymers. MDPI AG; 2016 [consultado: 24 enero 2021]. Disponible en: / pmc/articles/PMC6432532/?report=abstract

[32] Gregor A, Filová E, Novák M, Kronek J, Chlup H, Buzgo M, et al. Designing of PLA scaffolds for bone tissue replacement fabricated by ordinary commercial 3D printer. J Biol Eng. 2017 Oct 16;11(1) [consultado: 3 diciembre 2020]. Disponible en: /pmc/articles/ PMC5641988/?report=abstract

[33] Serra T, Planell JA, Navarro M. High-resolution PLA-based composite scaffolds via 3-D printing technology. Acta Biomater. 2013 Mar 1;9(3):5521-30.

[34] Ikada Y. Challenges in tissue engineering. J R Soc Interface. 2006 Oct 22;3(10):589-601 [consultado: 24 enero 2021]. Disponible en: https://royalsocietypublishing.org/doi/10.1098/ rsif.2006.0124

[35] Lee CH, Cook JL, Mendelson A, Moioli EK, Yao H, Mao JJ. Regeneration of the articular surface of the rabbit synovial joint by cell homing: A proof of concept study. Lancet. 2010 Aug 7;376(9739):440-8.

[36] Serrano-Aroca Á, Vera-Donoso CD, Moreno-Manzano V. Bioengineering approaches for bladder regeneration. Vol. 19, International Journal of Molecular Sciences. 2018, p. 1796.

[37] Abreu E. Scaffolding in Tissue Engineering. Biomed Eng Online. 2006 Dec;5(1):52 [consultado: 22 diciembre 2020]. Disponible en: https://www.ncbi.nlm.nih.gov/pmc/articles/PMC1594575/

[38] Gioumouxouzis CI, Karavasili C, Fatouros DG. Recent advances in pharmaceutical dosage forms and devices using additive manufacturing technologies. Vol. 24, Drug Discovery Today. Elsevier Ltd; 2019, pp. 636-43. 
[39] Khaled SA, Burley JC, Alexander MR, Yang J, Roberts CJ. 3D printing of tablets containing multiple drugs with defined release profiles. Int J Pharm. 2015 Oct 30;494(2):643-50.

[40] Murphy S V., Atala A. 3D bioprinting of tissues and organs. Vol. 32, Nature Biotechnology. Nature Publishing Group; 2014, pp. 773-85 [consultado: 12 febrero 2021]. Disponible en: https:// www.nature.com/articles/nbt.2958

[41] Lee W, Pinckney J, Lee V, Lee JH, Fischer K, Polio S, et al. Three-dimensional bioprinting of rat embryonic neural cells. Neuroreport. 2009 May 27;20(8):798-803 [consultado: 24 enero 2021]. Disponible en: https://pubmed.ncbi.nlm.nih.gov/19369905/

[42] Ali M, PR AK, Yoo JJ, Zahran F, Atala A, Lee SJ. A Photo-Crosslinkable Kidney ECM-Derived Bioink Accelerates Renal Tissue Formation. Adv Healthc Mater. 2019 Apr 6;8(7):1800992 [consultado: 24 enero 2021]. Disponible en: https://onlinelibrary.wiley.com/doi/abs/10.1002/ adhm.201800992

[43] Kim JH, Seol YJ, Ko IK, Kang HW, Lee YK, Yoo JJ, et al. 3D Bioprinted Human Skeletal Muscle Constructs for Muscle Function Restoration. Sci Rep. 2018 Dec 1;8(1) [consultado: 24 enero 2021]. Disponible en: https://pubmed.ncbi.nlm.nih.gov/30120282/

[44] Skardal A, Devarasetty M, Kang HW, Seol YJ, Forsythe SD, Bishop C, et al. Bioprinting cellularized constructs using a tissue-specific hydrogel bioink. J Vis Exp. 2016 Apr 1;2016(110) [consultado: 24 enero 2021]. Disponible en:/pmc/articles/PMC4941985/?report=abstract

[45] Liu K, Zhang Q, Li X, Zhao C, Quan X, Zhao R, et al. Preliminary application of a multilevel 3D printing drill guide template for pedicle screw placement in severe and rigid scoliosis. Eur Spine J. 2017 Jun 1;26(6):1684-9 [consultado: 24 enero 2021]. Disponible en: https://ink. springer.com/article/10.1007/s00586-016-4926-1

[46] George M, Aroom KR, Hawes HG, Gill BS, Love J. 3D Printed Surgical Instruments: The Design and Fabrication Process. World J Surg. 2017 Jan 1;41(1):314-9.

[47] Rankin TM, Giovinco NA, Cucher DJ, Watts G, Hurwitz B, Armstrong DG. Three-dimensional printing surgical instruments: Are we there yet? J Surg Res. 2014 Jun 15;189(2):193-7. 